\section{Photoacoustic technique for assessing optical scattering properties of turbid media}

\author{
Janaka C. Ranasinghesagara, Yan Jian, Xuhui Chen, \\ Kory Mathewson, and Roger J. Zemp \\ University of Alberta, Department of Electrical and \\ Computer Engineering, Edmonton, Alberta T6G 2V4, Canada
}

\begin{abstract}
Measurement of tissue optical properties impacts both optical diagnostic and theraputic applications. Although existing photoacoustic imaging techniques provide optical absorption contrast, we present a photoacoustic technique that demonstrates sensitivity to the optical scattering coefficient of a turbid medium. By incrementing the distance between a surface illumination spot and a subsurface absorber and measuring the photoacoustic amplitude of the absorber, we can effectively estimate the Green's function of light transport in a turbid medium. Our results for different concentrations of Intralipid indicate that this technique has the ability to distinguish small changes of the reduced scattering coefficient. It has the potential to be used for in vivo studies to obtain reduced scattering coefficients of biological tissues. These findings will potentially improve the calculation of subcutaneous fluence in photoacoustic-based techniques and laser dosimetry studies in live tissues. () 2009 Society of Photo-Optical Instrumentation Engineers. [DOI: 10.1117/1.3200922]
\end{abstract}

Keywords: optical scattering; photoacoustic imaging; high-frequency ultrasound; Q-switched lasers; biophotonics; index matching liquid.

Paper 09168LR received Apr. 29, 2009; revised manuscript received Jun. 18, 2009; accepted for publication Jun. 23, 2009; published online Aug. 7, 2009.

Optical properties of biological tissues are important in various optical diagnostic techniques and laser dosimetry calculations. ${ }^{1,2}$ Additionally, quantitative estimation of the scattering coefficient of tissue will improve our ability to estimate blood oxygenation and tissue oxygen consumption noninvasively, which will impact diagnosis and treatment of many different diseases. ${ }^{3}$ Previous methods developed to measure optical properties can be divided into two classes: direct and indirect. ${ }^{1}$ In direct techniques, Beer's law is applied on a thin sample by ignoring multiple scattered photons. This method requires removing a thin slice of a tissue for the measurement. It is not practical for certain media and cannot be used for in vivo measurements. However, this limitation is eliminated in indirect measurements. When optical reflectance or transmittance measurements are acquired, theoretical models such as radiative transport theory or diffusion theory are applied to calculate optical parameters. In commonly used techniques, normal incident reflectometry (NIR) ${ }^{4}$ and obliqueincident reflectometry $(\mathrm{OIR})^{3}$, one has to image or scan a

Address all correspondence to: Roger J. Zemp, Department of Electrical and Computer Engineering, University of Alberta, Edmonton, Alberta T6G 2V4, Canada. Tel: 780-492-1825; Fax: 780-492-1811; E-mail: zemp@ece.ualberta.ca relatively large tissue area to obtain accurate measurement because those methods are based on spatial distribution of diffuse reflectance. In addition, the surface condition of the tissue and detection apparatus may have an effect on the reflectance measurement. ${ }^{1}$

In the last decade, there has been tremendous interest in photoacoustic imaging of soft tissues. ${ }^{5,6}$ When short laser pulses are fired into a tissue, acoustic pressure waves are generated from optical absorbers due to thermoelastic expansion. The acoustic pressure wave or photoacoustic signal that is related to locally absorbed laser energy of an optical absorbing structure can be detected by an ultrasound transducer. Spatial resolution of the photoacoustic image is determined by the ultrasound focus and receiver bandwidth. This photoacoustic signal is directly proportional to optical absorption, laser fluence, and the Gruneisen parameter. The local laser fluence at the absorber location is determined by a combination of optical absorption and optical scattering. Current multiwavelength photoacoustic methods of blood oxygen saturation estimation suffer from unknown laser fluences at subsurface vessel. By knowing the wavelength-dependent tissue optical scattering properties, laser fluence at the blood vessel locations may be estimated more accurately to improve $\mathrm{SO}_{2}$ estimates. In this letter, we propose a photoacoustic technique for assessing optical scattering properties of tissues. In contrast to surface reflectance measurements in NIR and OIR techniques, this method measures the effective fluence at an absorber located inside the tissue.

In this technique, we focus a small laser spot on to the surface of a turbid medium. We use a focused ultrasound transducer to record the photoacoustic signal due to a subsurface absorbing structure. The fluence at the absorbing structure depends on the optical properties of the turbid medium. While keeping the ultrasound focus on the fixed absorbing structure, the light spot is laterally translated across the surface. The fluence at the absorbing structure changes with the distance between the light spot and the absorbing structure. The resultant photoacoustic signal captured by the transducer is a function of light-spot translation distance, and the measured curves of photoacoustic amplitude versus light-spot translation distance provide an estimate of the Green's function of light transport, because the photoacoustic signal is proportional to local laser fluence.

We developed a unique photoacoustic probe for this purpose. Figure 1 illustrates the design of the probe. It consists of two focusing lenses ( $\mathrm{f}=25$ and $40 \mathrm{~mm}$ ), a 10-mm fused silica prism, a 25-MHz ultrasound transducer (V324-SM, Panametrics-NDT, Waltham, MA, USA), and an indexmatching liquid (Catalog No. 19569, Cargille Labs, Cedar Grove, New Jersey). The transducer has a 6-mm active aperture and a 12.7-mm focal distance. The index-matching liquid has a refractive index similar to fused silica $(n=1.46)$. The prism, the index-matching liquid, and the ultrasound transducer are housed in a custom-machined acrylic holder. The index-matching fluid was held underneath the prism by attaching a $\sim 25$ - $\mu$ m-thick Saran Wrap membrane to the bottom of the holder using double-sided tape. The focusing lenses above the prism were mounted separately so that it can

1083-3668/2009/14(4)/040504/3/\$25.00 @ 2009 SPIE 


\section{JBO LETTERS}

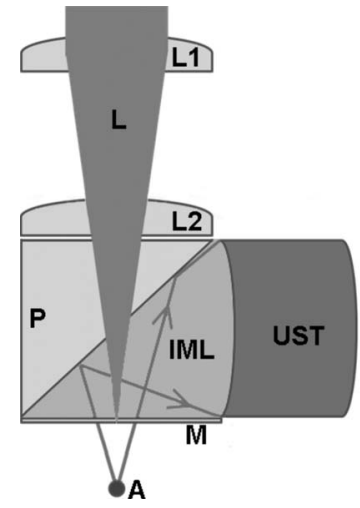

(a)

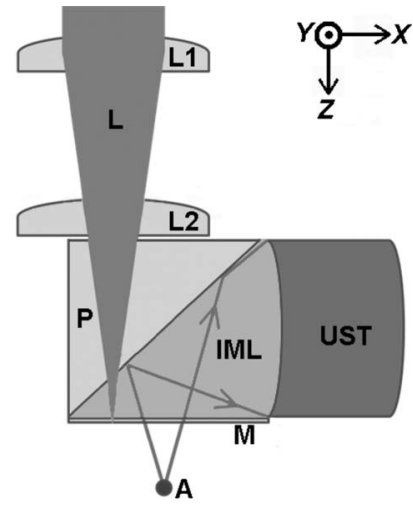

(b)
Fig. 1 Photoacoustic probe. L1: lens $f=25 \mathrm{~mm}$, optional L2: lens $f$ $=40 \mathrm{~mm}$ with radius of curvature $=$ distance between $\mathrm{L} 2$ surface and optical focus, L: pulsed laser beam, P: $10 \mathrm{~mm}$ right-angle quartz silica prism, IML: index-matching liquid, UST: ultrasound transducer, $M$ : fine membrane, A: Absorbing structure located in a turbid media. (a) Laser beam confocal with the ultrasound focus and (b) laser beam after translocation.

be translated along $z$-axis to form a small focused laser spot at the surface of the turbid medium.

The photoacoustic signal propagating up into the indexmatching liquid medium is reflected at the interface to reach the horizontally mounted ultrasound transducer. The maximum photoacoustic signal is produced when the ultrasound focus is confocal with the laser beam [Fig 1(a)]. A human hair attached to a thin plastic plate with a $4 \times 2-\mathrm{cm}$ opening was mounted parallel to the $y$-axis. It was aligned with the ultrasound focus via an $X Y Z$ stage by maximizing the ultrasound pulse echo signal.

The optical index-matching liquid enables nonrefracted light delivery through the fused silica prism to the turbid medium while providing a suitable propagation medium for the photoacoustic signal. The acoustic impedance and acoustic speed values are close to that of soft tissue or water, fortuitously minimizing reflections or distortions at the liquidtissue boundary (Table 1). Only $1 \%$ intensity reflection coefficients for normal incidence ultrasound are expected. Additionally, some minimal losses due to the fine membrane will occur.

Table 1 Optical and acoustic properties of fused silica, index matching liquid, and water. Subscripts $L$ and $T$ represent the longitudinal and transverse acoustic waves in the fused silica.

\begin{tabular}{lcccc}
\hline & $\begin{array}{c}\text { Refractive } \\
\text { index }\end{array}$ & $\begin{array}{c}\text { Acoustic } \\
\text { impedance } \\
(\text { MRayl })\end{array}$ & $\begin{array}{c}\text { Speed of } \\
\text { sound } \\
(\mathrm{mm} / \mu \mathrm{s})\end{array}$ & $\begin{array}{c}\text { Attenuation } \\
(\mathrm{dB} / \mathrm{cm} \\
\text { at } 25 \mathrm{MHz})\end{array}$ \\
\hline Fused silica & 1.46 & $Z_{\mathrm{L}}=12.9$ & $\mathrm{c}_{\mathrm{L}}=5.848$ & 0.006 \\
& & $Z_{T}=8.1$ & $c_{T}=3.687$ & \\
Index matching & 1.46 & $1.20 \pm 0.003$ & $1.44 \pm 0.04$ & $2.7 \pm 0.1$ \\
liquid & 1.33 & $1.51 \pm 0.003$ & $1.51 \pm 0.03$ & $1.38 \pm 0.02$ \\
Water & & & &
\end{tabular}

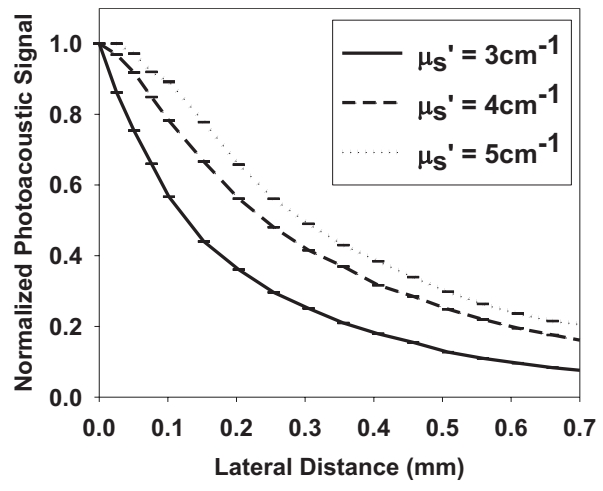

Fig. 2 Normalized photoacoustic signal as a function of lateral translocation distance for different $\mu_{\mathrm{s}}^{\prime}$. Error bars represent the standard deviation of 100 measurements.

Because the acoustic impedances of the optical indexmatching liquid and fused silica glass are quite different, the optimal reflection of acoustic energy can be attained at the glass-liquid interface. Our calculation shows that when the ultrasound incident angle (with respect to normal of the prism surface) is $>23 \mathrm{deg}, 100 \%$ of ultrasound will be reflected. The ultrasound incident angle at the glass-liquid interface is $45 \pm 13.4 \mathrm{deg}$; hence, all of the photoacoustic signal will be reflected toward the transducer. The speed of sound in the index-matching liquid is close to the speed of sound in water. The measured acoustic attenuation in the index matching liquid is $2.7 \pm 0.1 \mathrm{~dB} / \mathrm{cm}$ at $25 \mathrm{MHz}$, only slightly higher than water.

Initially, we used pulse echo ultrasound to position the target absorber (the hair) at the ultrasound focus by maximizing the output signal that was first amplified by an ultrasound pulse receiver (5073PR, Panametrics-NDT, Waltham, Massachusetts) and then captured by a digital oscilloscope (DPO7054, Tektronix, Beaverton, Oregon). After receiving the maximum signal, the translation stage was locked and the ultrasound receiver was changed to receive mode. A horizontal laser beam $(532 \mathrm{~nm}, \mathrm{Nd}: \mathrm{YAG})$ deflected downward by a prism and lens was attached to an $X Y Z$ stage. The laser beam was focused on to the fine membrane by translating the lens along the $z$-axis. The optical focus was set slightly below the membrane surface. The prism and lens were translated along the $x$-axis until the maximum photoacoustic signal was observed. At this stage, the laser beam and ultrasound focus are confocal. In addition to a strong photoacoustic signal from the hair, we observed a weak photoacoustic signal from the fine membrane. The depth of the human hair was determined by obtaining the time delay between the photoacoustic signal of the human hair and the fine membrane. The measured depth from the fine membrane to the human hair was $2.05 \mathrm{~mm}$.

As the reduced scattering coefficient $\mu_{\mathrm{s}}^{\prime}$ for Intralipid $\circledast$ varies from batch to batch, we employed an oblique-incidence diffuse reflectance technique ${ }^{3}$ to calculate $\mu_{\mathrm{s}}^{\prime}$ at $532 \mathrm{~nm}$ for our stock. We added known Intralipid concentration to water in the tank to produce a predetermined $\mu_{\mathrm{s}}^{\prime}$ value. Figure 2 illustrates the normalized photoacoustic signal as a function of lateral light-spot translocation distance for various $\mu_{\mathrm{s}}^{\prime}$. It can be seen that this method shows clearly separable curves for different $\mu_{\mathrm{s}}^{\prime}$ values. As $\mu_{\mathrm{s}}^{\prime}$ increases, curves get closer to each other. We demonstrated that our technique is capable of dis- 
tinguishing changes of $\mu_{\mathrm{s}}^{\prime}$ in increments of $1 \mathrm{~cm}^{-1}$, however, considering the small error bars for measured points, much finer resolution may be possible, and will be signal-to-noise dependent. Although we used $532 \mathrm{~nm}$ for this study due to laser availability, the $\mu_{\mathrm{s}}^{\prime}$ values we used in this experiment are smaller than the $\mu_{\mathrm{s}}^{\prime}$ values for the majority of biological tissues at that wavelength. But in the near-infrared region, $\mu_{\mathrm{s}}^{\prime}$ values of biological tissues are smaller and close to the values we used in the experiment. ${ }^{7}$

Because the ultrasound transducer focus is $12.7 \mathrm{~mm}$, the maximum depth between the membrane and the absorber is limited $(<3 \mathrm{~mm})$ by design. Light transport in this region is given by the radiative transport equation. We need transportregime analytical models or Monte Carlo simulations to estimate optical parameters from these curves. For target depths beyond 3-4 $\mathrm{mm}$, we will be able to use analytical equations based on the diffusion approximation. ${ }^{8}$ In future studies, we will employ an appropriate method to extract optical properties from the above curves.

To obtain a strong photoacoustic signal, we used a fraction of a millijoule pulse energy over $\sim 200$ - $\mu$ m-diam focused light spot. The estimated fluence of $\sim 1 \mathrm{~J} / \mathrm{cm}^{2}$ at the surface is above the ANSI exposure limitations. Several alternatives could be used to spread the laser fluences over a broad area to satisfy the ANSI limits. Instead of using a single high-energy pulse, we could use a series of pulses with low laser energy and average the signals. Another possibility is to use a ring illumination pattern such that the center of the ring will be coaligned with the absorbing structure. Otherwise, the size of the laser-ocusing spot can be increased to spread the laser fluence over a broader range, at the expense of blurring the effective Green's functions. This, however, may be acceptable as long as optical properties can still be estimated.

In this technique, the laser spot was translated only a short distance along the $x$-axis $(<1 \mathrm{~mm})$ to get the normalized photoacoustic signal. Hence, this method is suitable for samples with a small surface area. That is a great advantage over existing NIR and OIR methods. Even though this method presently uses only phantoms, it has the potential to be used for in vivo studies, where a small blood vessel can be isolated as an absorbing structure. However, more blood vessels (absorbing structures) present in the region (spaced closer than an acoustic focal width) may adversely affect the results.

To date, we know of no method that measures the internal fluence of a turbid medium to obtain its optical parameters. The measured curves can be considered an estimate of the normalized Green's function of light transport. Fitting the measured curves to models of light transport may facilitate estimation of the scattering and absorption coefficients of tissue. Such predictions may improve existing estimates of absolute absorber concentrations in photoacoustic imaging, including photoacoustic-based oxygen saturation imaging and laser dosimetry. Our current study is limited to an isotropic turbid medium with a single laser source. Speed-of-sound inhomogeneities will lead to receive-focusing aberrations that may introduce a source of variance in the measured scattering curves, although this is estimated to be a minor effect over the small tissue volumes interrogated. Optical heterogeneities will also be a source of error; however, we may be able to obtain optical parameters of complex tissue structures with multiple sources and detectors. Future work may include inverse prob- lems attempting to estimate absorption and scattering inhomogeneities due to multiple optical sources and photoacoustic detection.

In summary, we described a novel photoacoustic concept to differentiate turbid media with different scattering coefficients. In previous attempts of using photoacoustic methods to demonstrate sensitivity to optical scattering,,${ }^{9,10}$ an ultrasound transducer was mounted on the side of a cuvette or opposite the side of the light delivery. Such a setup is not feasible for measuring an optical scattering coefficient of live tissues. This is the first report to our knowledge of using photoacoustic methods that can be applied to demonstrate sensitivity to optical scattering in the reflection mode. We developed a unique probe to implement the above concept, which allows unparalleled flexibility in photoacoustic light delivery. The results obtained for different concentrations of Intralipid clearly demonstrate that this technique can be employed to distinguish small changes in the value of $\mu_{\mathrm{s}}^{\prime}$. In future studies, we will compare these curves with light-transport models to obtain optical properties of the turbid medium. Such findings have the potential to quantitatively image and estimate tissue properties.

We sincerely acknowledge Dr. Robert Fedosejevs for sharing his Nd:YAG laser with our group. This work was sponsored by the Terry Fox Foundation through the National Cancer Institute of Canada (Grants No. NCIC TFF 019237 and No. 019249), NSERC (Grant No. G121211115), the Alberta Cancer Board (Grant No. ACB 23728), Canada Foundation for Innovation Leaders Opportunity Fund, Alberta Advanced Education \& Technology, and the University of Alberta for equipment and infrastructure.

\section{References}

1. W. F. Cheong, S. A. Prahl, and A. J. Welch, "A review of the optical properties of biological tissues," IEEE J. Quantum Electron. 26, 2166-2185, (1990)

2. S. A. Prahl, M. J. C. van Gemert, and A. J. Welch, "Determining the optical properties of turbid media by using the adding-doubling method," Appl. Opt. 32, 559-568 (1993).

3. S.-P. Lin, L. V. Wang, S. L. Jacques, and F. K. Tittel, "Measurement of tissue optical properties using oblique incidence optical fiber reflectometry," Appl. Opt. 36, 136-143 (1997).

4. T. J. Farrell, M. S. Patterson, and B. Wilson, "A diffusion-theory model of spatially resolved, steady-state diffuse reflectance for the noninvasive determination of tissue optical-properties in vivo," Med. Phys. 19, 879-888 (1992).

5. C. G. A. Hoelen, F. F. M. de Mul, R. Pongers, and A. Dekker, "Threedimensional photoacoustic imaging of blood vessels in tissue," Opt. Lett. 23, 648-650 (1998).

6. X. D. Wang, Y. J. Pang, G. Ku, X. Y. Xie, G. Stoica, and L. H. V. Wang, "Noninvasive laser-induced photoacoustic tomography for structural and functional in vivo imaging of the brain," Nat. Biotechnol. 21, 803-806 (2003).

7. M. Lualdi, A. Colombo, B. Farina, S. Tomatis, and R. Marchesini, "A phantom with tissue-like optical properties in the visible and near infrared for use in photomedicine," Lasers Surg. Med. 28, 237-243 (2001).

8. R. J. Zemp, J. Ranasinghesagara, Y. Liang, X. Chen, and K. Mathewson, "A photoacoustic method for optical scattering measurements in turbid media," Proc. SPIE 7177, 71770Q (2009).

9. A. A. Oraevsky, S. L. Jacques, and F. K. Tittel, "Measurement of tissue optical properties by time-resolved detection of laser-induced transient stress," Appl. Opt. 36, 402-415 (1997).

10. Z. Zhao and R. Myllylä, "Measuring the optical parameters of weakly absorbing, highly turbid suspensions by a new technique: photoacoustic detection of scattered light," Appl. Opt. 44, 7845-7852 (2005). 